\title{
Wuhan Bridge Group Monitoring Networking Platform Based on Health Monitoring and Inspection
}

\author{
Tian Xia ${ }^{1}$, Jinghua $\mathrm{Fu}^{*}{ }^{2}$, a , Xiaochuan $\mathrm{He}^{1, \mathrm{~b}}$, Xiao Zhou ${ }^{1}$, Chen Xiao ${ }^{1}$ and \\ Xiaoyong Huang ${ }^{1}$ \\ ${ }^{1}$ Wuhan Roads \& Bridges Toll Management Center, Wuhan, China \\ ${ }^{2}$ National Engineering Laboratory for Fiber Optic Sensing Technology, Wuhan Univ. of Technology, \\ Wuhan, China \\ a foryoufirst@163.com, b15572715@qq.com
}

Keywords: bridge group, network monitoring, health monitoring, inspection

Abstract. There are some bridges in Wuhan which had independently equipped with safety monitoring system, and bridge management is mainly manual work. By building the bridge group network monitoring platform can effectively integrate the former bridge monitoring system, at the same time; the platform can give consideration to the information and digitization work of daily inspection and maintenance. It can also change the current business model of bridge safety management and enhance scientific and digitization management of conventional work of bridge.

\section{Introduction}

The geographical pattern of Wuhan city determines that most of bridges across the Yangtze River and Hanjiang River in nearly 100 bridges which play an important role in the construction of Wuhan city circle and the public travel. At present, although many large bridges in Wuhan have been equipped with a safety monitoring system or are being put into practice, the existence of bridge monitoring projects still have some differences in implement time, contents, objectives and standards, not comprehensive in monitoring content and not standardization in monitoring module division. And do not form a unified concept of network management; safety monitoring system of each bridge is stand-alone, the information is not shared interaction and there is a certain part of defects. Safety monitoring system for bridge and other management system (such as security monitoring, power monitoring and weighing system) are independent of each other and information is not fusion[1,2].

According to the characteristics of super large bridge, as the manager, Wuhan bridge toll management center establish bridge maintenance manual[3,4] which takes "Prevention first, combining prevention with control, combining routine maintenance and general repair" as the guideline that can manage the disease and risk of the bridge and restore or improve the carrying capacity of the bridge structure. In ensuring the safe operation while maximum extends the service life of bridge and gives full play to the function of bridge. Currently, bridge management in Wuhan is mainly based labor work. The record of daily inspection, regular inspection and outsourcing inspection is by hand, and the bridge daily management data also for paper file, which bring a lot of inconvenience for bridge maintenance.

Therefore, the construction of the internet of bridges platform as an opportunity can integrate each former bridge monitoring system, while taking into account the current digital and information work of bridge daily inspection, repair and maintenance. This platform not only can guide the future construction of bridge health and safety monitoring system in Wuhan, but also improve the current scientific and digital management level of bridge routine work. All in all, building and construction by stages of the internet of bridges platform is significant which can gradually refine the formation of industry standards and support the construction of smart city of Wuhan. 


\section{Platform construction target}

The internet of bridges platform can make the structural safety monitoring system and bridge toll management center OA system to form an organic whole, and in the demand for service-oriented architecture, it realizes unified accession of data between structure safety monitoring and office management OA system and also realizes data fusion of subsystem which can reduce the redundant data record. By the inspection results of the sensor monitoring with digitization, automation and visualization, traditional manual visual inspection and professional inspection team, the platform can afford detailed preliminary conclusions for security condition of bridge regularly and meet the daily office needs for bridge manager at all levels and help bridge expert to make an evaluation for bridges.

\section{Platform overall architecture}

Wuhan Yangtze river second bridge and Baishazhou Yangtze River Bridge are the first phase in the construction of the internet of bridges in Wuhan. Ensuring structure safety and scientific guidance for bridge management are the targets in the platform which include structure monitoring, inspection, the office OA system of bridge toll management center and other main information modules. The representation of information is mainly based on the three dimension simulation model of bridge, and with all kinds of monitoring curve and report. Equipment fault diagnosis module is gradually improving by design; the expert system of the platform will realize the maximum difference between equipment failure and structure monitoring alarm. With the help of three dimension simulation model of bridge, the information of structure safety and bridge diseases will be intuitive visual display. Through the background algorithm to form the predefined database, monitoring data can provide the relevance, trends and statistics of all kinds of information for the structure safety analysis. In addition, in view of the management operation records, main equipment running status, as shown in Figure 1.

\section{Hardware environment of internet data center}

Establish data center hardware environment in bridge toll management center while construction corporative backup hardware environment in Wuhan University of technology. This double line platform is responsible for distributed bridge terminal to centralized center for integrated management of enterprise-level application model, which can realize centralized management for data, software services, system security, upgrade and maintenance, and make new bridges to access conveniently. Any access to the system need to pass data center portal security certification, the new system (service) to join and use all need portal security certification.

\section{System construction of internet data center software}

System construction of internet data center software is the central storage and control centers of the bridge health monitoring subsystem. The responsibility of the system is to centralize collection, management, analysis, calculation and storage for each bridge site data, and includes subsystem security permissions for each bridge unified management and control. The system includes the following components.

1)Security permissions and unified management system

System adopts B/S structure and deploys in the data center server. It is responsible for system management and unified management of security permissions for each subsystem. Using the support system can realize general network by once login and avoid repetition login. The construction of main function modules include: application system management, role management, user management, rights management, service to add and delete, service dispatch, and the integration framework.

2) management software of internet data center

Management Station software installed in the data center server which receive, summarize and store collection data of bridges. In addition to deal with the monitoring data, the receive station is also 


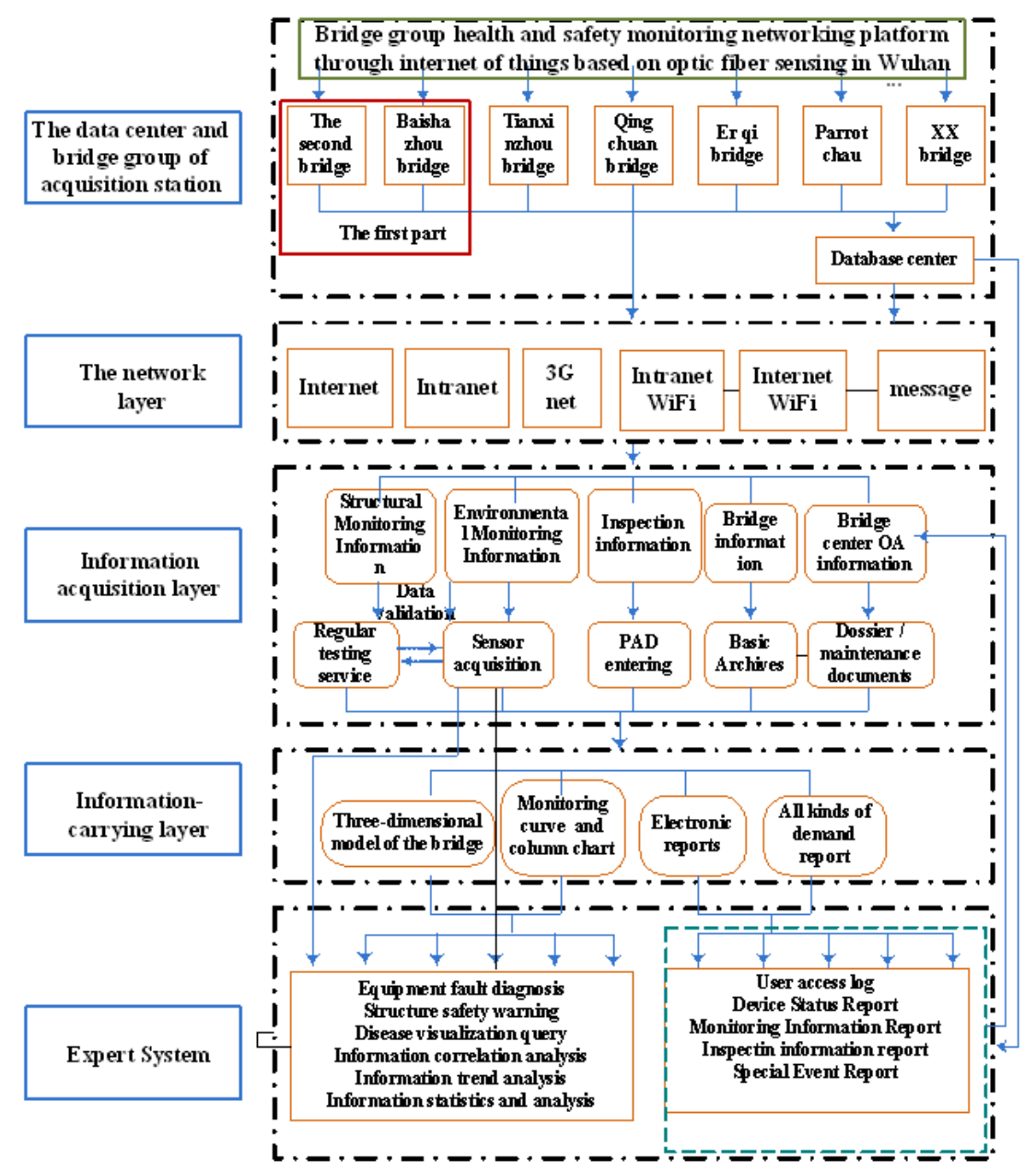

Figure1 The overall deployment diagram

responsible for receiving digital tablet input data by manual inspection. The construction of main function modules include: the bridge terminal sensing data receiving, data communication, the server data storage, data statistic and analysis, comprehensive diagnosis system.

3) monitoring display software of internet data center

The bridge site monitoring center deployment of single bridge health monitoring software is used to display the local LAN monitoring, and the monitoring display software on the internet data center shows all the access bridge health monitoring subsystem information together, which can switch to show each bridge monitoring, provide a bridge monitoring data comparison function, as well as provide a platform for integrated services that include disease three-dimensional query service, manual inspection information service, bridge file service, structure safety monitoring service, video monitoring service, office OA service, information syndication service, leadership inquiry service, technical condition evaluation service. The data source of the subsystem comes from the centralized data center server which provides public access function, as shown in Figure 2.
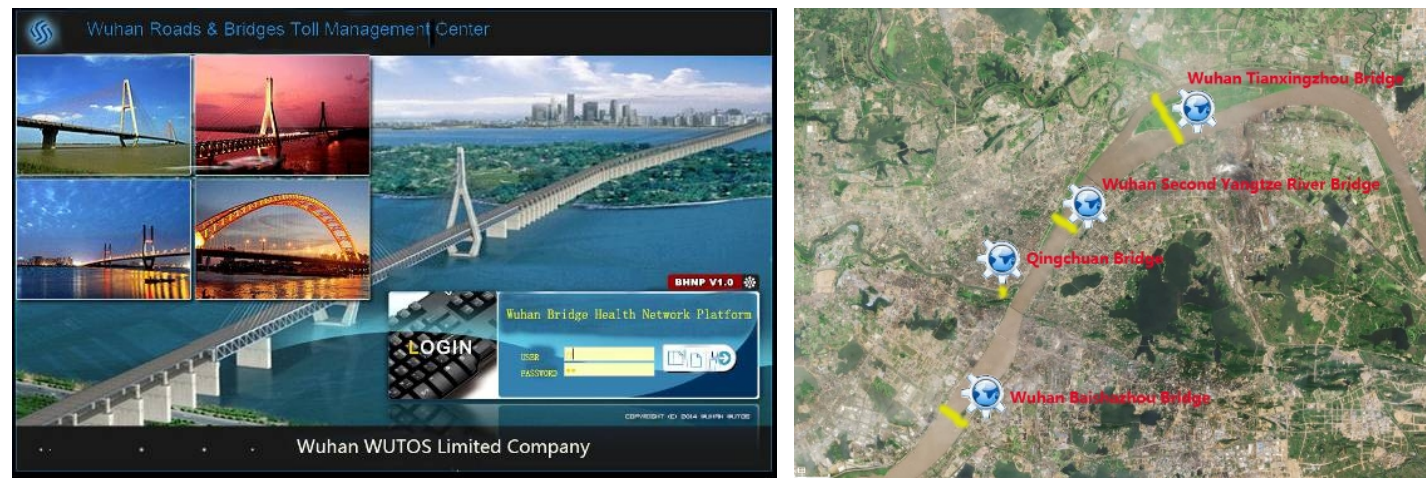

Figure2 the interface of the internet of bridges platform software 
4) Digital inspection and integrated management software

Digital inspection and integrated management software through a centralized inspection management subsystem to manage and analyze data which can make a decision for maintenance. Combined with some specifications and maintenance manuals of bridges [5, 6], development of bridge inspection information input system on handheld mobile terminal. By using the mobile intelligent terminal to complete the inspection information collecting and recording in the bridge site, and then sends the data to the monitoring center server through network, the internet data center management software system can make a management and evaluation for inspection data(shown in Figure 3,4).

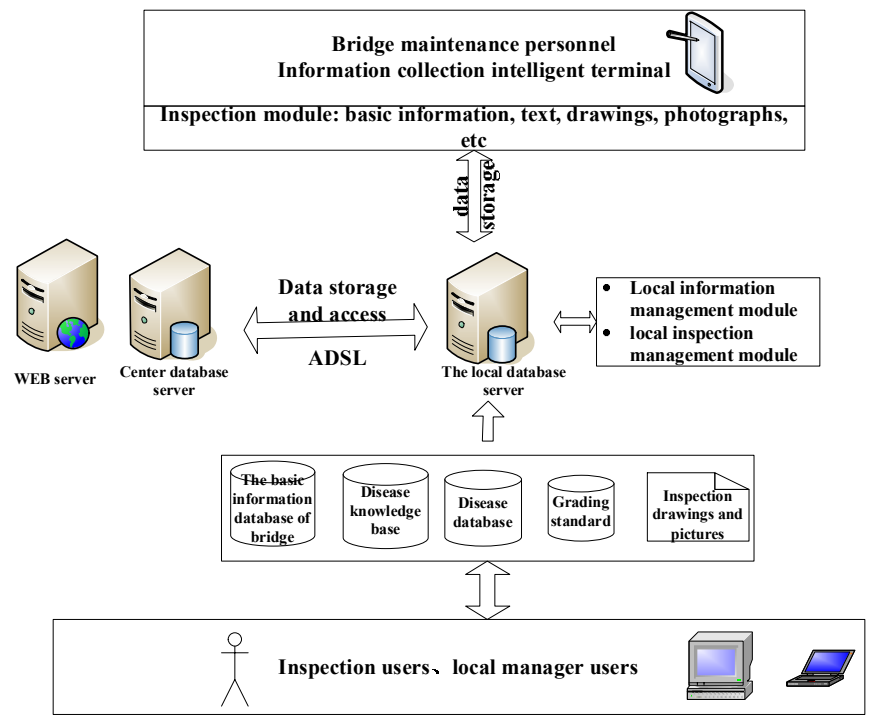

Figure3 management and receive of inspection data

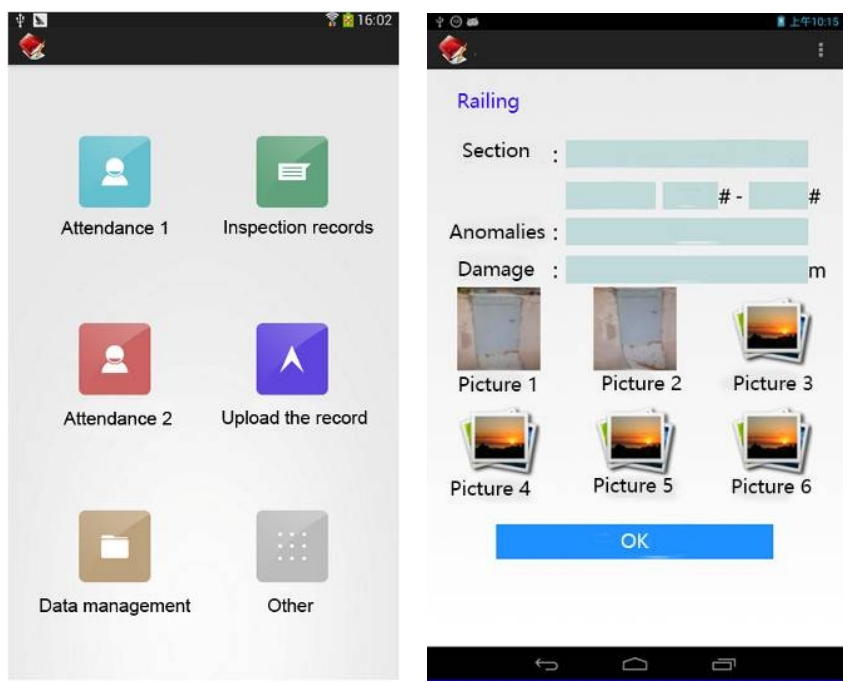

Figure4 the interface of bridge inspection information input system

\section{Conclusion}

The construction of the internet of bridges platform as an opportunity can integrate each former bridge monitoring system and combine with digital and information work of bridge daily inspection, repair and maintenance, which can improve the scientific and digital management level and avoid duplication of investment and waste of resources, and can also realize information sharing and exchange, function with association and mutual assistance, operation and maintenance timely, safe and efficient management. In the process of construction of platform, form industry standards gradually and establish application model of the internet of bridges monitoring. 


\section{Acknowledgements}

This work was financially supported by the Fundamental Research Fund for the Central Universities(WUT:2014-IV-009, WUT: 2014-II-012).

\section{References}

[1] Wang Ying Jun, in: Research on the key technology of large cable-stayed bridge health monitoring system, edited by Wuhan University of technology, 2006.

[2] Wang JieZao, Zhong JiWei, Wang Bo, in: Designed components and development of health monitoring systems for long span bridges, edited by Bridge Construction (2009, S2)

[3] The Bridge Toll Management Center of Wuhan, china railway major bridge reconnaissance \& design institute co, ltd: Bridge maintenance and management manual -Wuhan Yangtze River Second Bridge Technical Volume (Wuhan, 2012).

[4] The Bridge Toll Management Center of Wuhan, china railway major bridge reconnaissance \& design institute co, 1td: Bridge maintenance and management manual -Wuhan BaiShaZhou Yangtze River Bridge Technical Volume (Wuhan, 2008).

[5] Ministry of Transport of the People's Republic of China: Code for Maintenance of Highway Bridges and Culvers edited by China Communications Press, Beijing, 2004.

[6] Ministry of Transport of the People's Republic of China: Standards for technical condition evaluation of highway bridges edited by China Communications Press, Beijing, 2004. 